\title{
AGM inequality with binomial expansion
}

\author{
Jamal Rooin* \\ Jamal Rooin received his M.S. from the Teacher Education University in Teheran, \\ Iran, in 1989. In 1997 he became a Ph.D. student at that university, where he grad- \\ uated in the year 2002. Since then he is an assistant professor at the Institute for \\ Advanced Studies in Basic Sciences in Zanjan, Iran. His mathematical research inter- \\ ests include the theory of inequalities, problems in analysis and functional analysis.
}

The classical Arithmetic mean-Geometric mean inequality, or briefly the AGM inequality, states that for any nonnegative real numbers $x_{1}, x_{2}, \ldots, x_{n}$, we have

$$
\sqrt[n]{x_{1} x_{2} \ldots x_{n}} \leq \frac{x_{1}+x_{2}+\cdots+x_{n}}{n}
$$

and equality occurs if and only if $x_{1}=x_{2}=\cdots=x_{n}$. There are several interesting proofs of the AGM inequality, see e.g. [1]-[4]. In this note, using the binomial expansion, we get a recursive relation between the successive differences of the arithmetic and geometric means, and then using it, we prove and sharpen the AGM inequality. All we need is the following lemma, in which

$$
A_{n}=\frac{x_{1}+x_{2}+\cdots+x_{n}}{n} \quad \text { and } \quad G_{n}=\sqrt[n]{x_{1} x_{2} \ldots x_{n}}
$$

are the standard notations for the arithmetic and the geometric means of $n$ given nonnegative numbers $x_{1}, x_{2}, \ldots, x_{n}$, respectively.

Ungleichungen spielen in der Mathematik bekanntlich eine wichtige Rolle. Die Ungleichung zwischen dem arithmetischen und geometrischen Mittel ist dabei von besonderem Interesse, da sie zu einer Vielzahl von weiteren Ungleichungen Anlass gibt. Es gibt mehrere interessante Beweise für diese Ungleichung; z.B. finden sich dazu über fünfzig Beweise mit historischen Kommentaren im Buch „Means and their inequalities“ von P.S. Bullen, D.S. Mitrinović und P.M. Vasić. In der vorliegenden Arbeit wird mit Hilfe des binomischen Lehrsatzes eine rekursive Beziehung zwischen den sukzessiven Differenzen von arithmetischem und geometrischem Mittel gegeben. Mittels vollständiger Induktion ergibt sich damit ein einfacher und eleganter Beweis für die Ungleichung zwischen dem arithmetischen und geometrischen Mittel.

*) The author is supported in part by the Institute for Advanced Studies in Basic Sciences, Zanjan, Iran. 
Lemma. With the above notations,

$$
A_{n}-G_{n}=\frac{1}{n} \sum_{k=2}^{n}\left(\begin{array}{l}
n \\
k
\end{array}\right) A_{n-1}^{\frac{n-k}{n}}\left(x_{n}^{1 / n}-A_{n-1}^{1 / n}\right)^{k}+x_{n}^{1 / n}\left(A_{n-1}^{\frac{n-1}{n}}-G_{n-1}^{\frac{n-1}{n}}\right) .
$$

Proof. By the binomial expansion, we have

$$
x_{n}=\left(x_{n}^{1 / n}-A_{n-1}^{1 / n}+A_{n-1}^{1 / n}\right)^{n}=\sum_{k=0}^{n}\left(\begin{array}{l}
n \\
k
\end{array}\right) A_{n-1}^{\frac{n-k}{n}}\left(x_{n}^{1 / n}-A_{n-1}^{1 / n}\right)^{k} .
$$

So,

$$
A_{n}=\frac{(n-1) A_{n-1}+x_{n}}{n}=A_{n-1}^{\frac{n-1}{n}} x_{n}^{1 / n}+\frac{1}{n} \sum_{k=2}^{n}\left(\begin{array}{l}
n \\
k
\end{array}\right) A_{n-1}^{\frac{n-k}{n}}\left(x_{n}^{1 / n}-A_{n-1}^{1 / n}\right)^{k}
$$

and therefore,

$$
\begin{aligned}
A_{n}-G_{n} & =A_{n}-G_{n-1}^{\frac{n-1}{n}} x_{n}^{1 / n} \\
& =A_{n}-A_{n-1}^{\frac{n-1}{n}} x_{n}^{1 / n}+x_{n}^{1 / n}\left(A_{n-1}^{\frac{n-1}{n}}-G_{n-1}^{\frac{n-1}{n}}\right) \\
& =\frac{1}{n} \sum_{k=2}^{n}\left(\begin{array}{l}
n \\
k
\end{array}\right) A_{n-1}^{\frac{n-k}{n}}\left(x_{n}^{1 / n}-A_{n-1}^{1 / n}\right)^{k}+x_{n}^{1 / n}\left(A_{n-1}^{\frac{n-1}{n}}-G_{n-1}^{\frac{n-1}{n}}\right) .
\end{aligned}
$$

Proof of the AGM inequality. Whithout loss of generality, we may suppose that $x_{1} \leq x_{2} \leq$ $\cdots \leq x_{n}$. So, by the fact that $x_{n} \geq A_{n-1}$ and the induction hypothesis $A_{n-1} \geq G_{n-1}$, we conclude that

$$
A_{n}-G_{n} \geq \frac{1}{n} \sum_{k=2}^{n}\left(\begin{array}{l}
n \\
k
\end{array}\right) A_{n-1}^{\frac{n-k}{n}}\left(x_{n}^{1 / n}-A_{n-1}^{1 / n}\right)^{k} \geq 0,
$$

and the AGM inequality is obtained.

For the case of equality in (1), it is evident from (2) that $A_{n}=G_{n}$ if and only if $x_{n}=A_{n-1}$ and $A_{n-1}=G_{n-1}$, which by the induction hypothesis is equivalent to $x_{1}=x_{2}=\cdots=x_{n}$.

\section{Remark}

(i) We can write

$$
\left(A_{n-1}^{\frac{n-1}{n}}-G_{n-1}^{\frac{n-1}{n}}\right) \sum_{l=0}^{n-1} A_{n-1}^{l / n} G_{n-1}^{\frac{n-1-1}{n}}=\left(A_{n-1}-G_{n-1}\right) \sum_{l=0}^{n-2} A_{n-1}^{l / n} G_{n-1}^{\frac{n-2-l}{n}},
$$

and so by (2),

$$
A_{n}-G_{n}=\frac{1}{n} \sum_{k=2}^{n}\left(\begin{array}{l}
n \\
k
\end{array}\right) A_{n-1}^{\frac{n-k}{n}}\left(x_{n}^{1 / n}-A_{n-1}^{1 / n}\right)^{k}+C_{n-1} x_{n}^{1 / n}\left(A_{n-1}-G_{n-1}\right),
$$


where

$$
C_{n-1}= \begin{cases}\frac{\sum_{l=0}^{n-2} A_{n-1}^{l / n} G_{n-1}^{\frac{n-2-1}{n}}}{\sum_{l=0}^{n-1} A_{n-1}^{l / n} G_{n-1}^{\frac{n-1-1}{n}}} & \text { if } G_{n-1} \neq A_{n-1}, \\ 0 & \text { if } G_{n-1}=A_{n-1},\end{cases}
$$

which is a recursive relation between successive differences of the arithmetic and the geometric means.

(ii) Using the mean value theorem for the function $f(x)=x^{\frac{n-1}{n}}$ over the interval $\left[G_{n-1}, A_{n-1}\right]$, there exists an $\xi_{n-1}$ with $G_{n-1} \leq \xi_{n-1} \leq A_{n-1}$, such that

$$
\xi_{n-1}^{1 / n}\left(A_{n-1}^{\frac{n-1}{n}}-G_{n-1}^{\frac{n-1}{n}}\right)=\frac{n-1}{n}\left(A_{n-1}-G_{n-1}\right) .
$$

Therefore, if $x_{n} \geq A_{n-1}$, we have $x_{n} \geq \xi_{n-1}$, and so by (2) and (5),

$$
A_{n}-G_{n} \geq \frac{1}{n} \sum_{k=2}^{n}\left(\begin{array}{l}
n \\
k
\end{array}\right) A_{n-1}^{\frac{n-k}{n}}\left(x_{n}^{1 / n}-A_{n-1}^{1 / n}\right)^{k}+\frac{n-1}{n}\left(A_{n-1}-G_{n-1}\right),
$$

which is a sharpening of Rado's inequality [2] for equal weights:

$$
A_{n}-G_{n} \geq \frac{n-1}{n}\left(A_{n-1}-G_{n-1}\right) .
$$

By a similar argument, if $x_{n} \leq G_{n-1}$, we see that the inequality in (6) reverses, and so, we obtain a converse of Rado's inequality.

(iii) If $0 \leq x_{1} \leq x_{2} \leq \cdots \leq x_{n}$, then considering (6) for $m$ instead of $n$, and then summing up (6) for $m=2, \ldots, n$, we have

$$
\begin{aligned}
n\left(A_{n}-G_{n}\right) & =\sum_{m=2}^{n}\left[m\left(A_{m}-G_{m}\right)-(m-1)\left(A_{m-1}-G_{m-1}\right)\right] \\
& \geq \sum_{m=2}^{n} \sum_{k=2}^{m}\left(\begin{array}{l}
m \\
k
\end{array}\right) A_{m-1}^{\frac{m-k}{m}}\left(x_{m}^{1 / m}-A_{m-1}^{1 / m}\right)^{k}
\end{aligned}
$$

which is a refinement of the AGM inequality. Similarly, if $x_{1} \geq x_{2} \geq \cdots \geq x_{n} \geq 0$, we see that the inequality in (7) reverses, and we get a converse of the AGM inequality.

\section{References}

[1] Beckenbach, E.F.; Bellman, R.: Inequalities. Springer, Berlin 1983.

[2] Bullen, P.S.; Mitrinović, D.S.; Vasić, P.M.: Means and Their Inequalities. Reidel, Dordrecht 1988.

[3] Hardy, G.H.; Littlewood, J.E.; Pólya, G.: Inequalities. Cambridge University Press, 1967.

[4] Mitrinović, D.S.: Analytic Inequalities. Springer, Berlin 1970.

Jamal Rooin

Institute for Advanced Studies in Basic Sciences

P.O. Box 45195-159

Zanjan, Iran

e-mail: Rooin@iasbs.ac.ir 